\title{
Population registration without the strawman, Polytopia
}

\author{
Johan Nygren \\ Panarchy Foundation
}

\begin{abstract}
The strawman paradigm has up until now been the only practical way to organize population registration. With the advent of new toolkits for legal interaction, such as digital signatures, mathematically verifiable digital ledger technology, and electronic communication over the internet, it is now possible to conceive of a new paradigm. This new paradigm, is the pseudonym events, global, simultaneous verification events that occur at the exact same time for every person on Earth.
\end{abstract}

\section{Introduction}

Population registration has traditionally relied on validating a person to a description of the person. While this mechanism has managed to provide one-person-one-personhood, it places a lot of trust in those attesting to the social proof. False attestations threaten to introduce false personhoods into the system. Imperfections in how the proof is selected also risks misrepresenting the individual, forming a "strawman".

The new paradigm substitutes the mechanism to validate a person to an arbitrary description, a strawman, with validating a person to a moment in time. It does this by validating every person in the population, at the exact same time, in global, simultaneous verification events. This new paradigm employs every single person in the validation of personhood, relying on digital signature technology to make that practical.

\section{Pseudonym events}

The current paradigm will be defined as the strawman paradigm, an arbitrary description is imposed on a person, and personhood is proven by validating the person to their strawman. The new paradigm, is the pseudonym events, global, simultaneous verification events that occur at the exact same time for every person on Earth (Ford, 2008). The paradigm shift is from proving unique personhood by "proof-of-strawman" to using "proof-of-pseudonym-event".

\section{Video interviews}

Within the new paradigm of Pseudonym events as a coordination mechanism instead of a strawman, the proposal to organize the verification by 1-on-1 video "Turing tests" (Nygren, 2018) seems to be game theoretically secure.

\section{Solving the stalemate}

To be able to do 1-on-1 video interviews, the stalemate problem required a solution. This solution was described by Johan Nygren in 2018, with the mechanism that other pairs can step in and judge a person if their pair has a stalemate. The standard mode of operation is 1-on-1, and 2-on-1 verification is only used if there is a problem.

\section{The "virtual border"}

The pseudonym parties also require a population with "human supremacy", to avoid fake accounts from outnumbering actual humans. This was achieved by requiring stricter verification when opting-in, creating a form of "border" or "wall" around it, described by Johan Nygren in 2018. In the 1-on-1 video implementation, using the same 2-on-1 "court" mechanism when a person first enters the population. 


\section{Randomization}

The randomization of the pairs is a major security assumption, and is very simple. The processes are separated over two phases. In the first phase, people register and are appended to a list. In the second phase, the list is shuffled using a standard fisher-yates shuffle, and, a randomly generated seed.

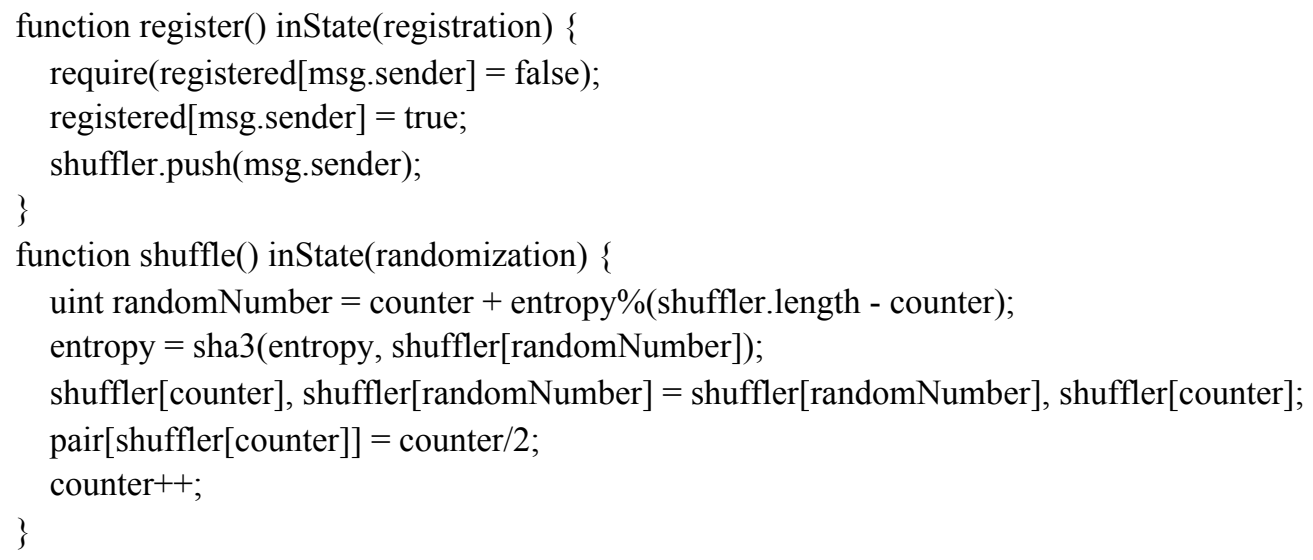

\section{Anonymity}

Since "who a person is" is not a factor in the proof, mixing of the proof-of-unique-human does not reduce the reliability of the protocol in any way. It is therefore allowed, and encouraged. This is practically achieved with "tokens" that are intermediary between verification in one pseudonym event and registration for the next event, authorizing mixer contracts to handle your "token" using the approve() function.

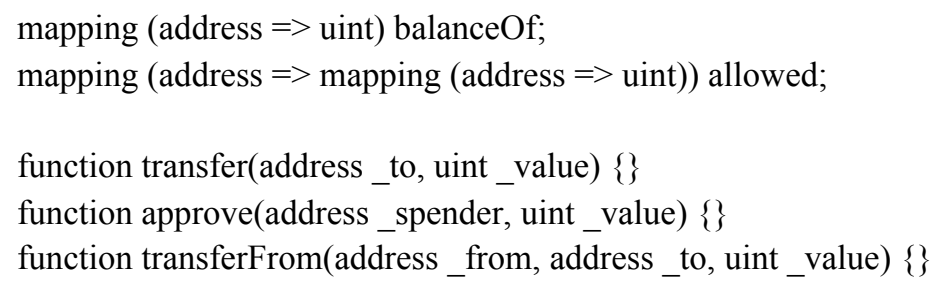

\section{Compartmentalization}

Since the proof is not based on who you are, and your proof-of-unique-human is untraceable from month to month, this means the system "resets" from month to month. The proofs, valid for a month, can be disposed of once no longer valid. The system operates completely compartmentalized from month to month. When compared to the traditional population registry system, people in Polytopia are legally "born" every month, and "die" every month.

\section{Collusion attacks}

Polytopia is vulnerable to one type of attack, collusion attacks. These are relatively harmless because the probability of gaining control of a pair is low. The exact success of collusion attacks is percentageColluding $\wedge 2$, it decreases with an inverse square relationship as the total colluding population decreases. For example, if $10 \%$ of the entire population colludes, they get $1 \%$ fake accounts. Another way to look at it, each attacker gets percentageColluding more than they would otherwise. Collusion attacks are allowed as they cannot be completely prevented. 


\section{Man-in-the-middle attacks}

Another attack vector is man in the middle attacks. These can be defended against perfectly, but require an extra step. The ideal defense adds a "handshake" to secure the video channel. The mechanism for this, the pair schedules a "pre-meeting" at a random time before the event, by agreeing on a random number using a commit-reveal scheme. Their channel can be proven secure by validating that they both got the same number. The probability of the man in the middle attacking this in a way that both peers get the same number is $1 /$ numberSize. This defense is as "Turing safe" (same difficulty for breaking Turing test) as the actual event itself.

\section{Implementation}

Implementation of Polytopia as a smart contract, a computer program running on digital ledger technology, is trivial.

\section{Scheduling}

Scheduling is trivial. The current month is calculated using a timestamp for the genesis event, the periodicity in seconds, and the current time.

uint constant period $=4$ weeks;

uint constant genesis $=198000$;

function schedule () returns (uint) \{ return genesis $+(($ block.timestamp - genesis $) /$ period $) *$ period; \}

\section{References}

Pseudonym Parties: An Offline Foundation for Online Accountable Pseudonyms, https://pdos.csail.mit.edu/papers/accountable-pseudonyms-socialnets08.pdf (2008)

Pseudonym Pairs: A foundation for proof-of-personhood in the web 3.0 jurisdiction, https://panarchy.app/PseudonymPairs.pdf (2018) 